\title{
PGC-1 coactivators: inducible regulators of energy metabolism in health and disease
}

\author{
Brian N. Finck ${ }^{1,2}$ and Daniel P. Kelly $y^{1,2,3,4}$
}

${ }^{1}$ Center for Cardiovascular Research, ${ }^{2}$ Department of Medicine, ${ }^{3}$ Department of Molecular Biology and Pharmacology, and ${ }^{4}$ Department of Pediatrics, Washington University School of Medicine, St. Louis, Missouri, USA.

\begin{abstract}
Members of the PPAR $\gamma$ coactivator-1 (PGC-1) family of transcriptional coactivators serve as inducible coregulators of nuclear receptors in the control of cellular energy metabolic pathways. This Review focuses on the biologic and physiologic functions of the PGC-1 coactivators, with particular emphasis on striated muscle, liver, and other organ systems relevant to common diseases such as diabetes and heart failure.
\end{abstract}

Members of the nuclear receptor (NR) superfamily relay physiologic and nutritional cues to critical gene regulatory responses. The molecular links between external stimuli, cellular signaling events, and NR-mediated transcriptional control are currently being unraveled. New information emerging over the past decade has demonstrated that NRs receive regulatory input through multiple mechanisms including levels of endogenous ligand, availability of heterodimeric NR partners, and posttranslational modifications. Activating signals trigger the recruitment of coactivator complexes onto the NR platform, leading to enzymatic modification of chromatin, increased access of the RNA polymerase II machinery to RNA, and activation of target gene transcription (Figure 1). Availability of certain coactivator proteins also serves critical regulatory functions linking physiologic stimuli to NR activity. Perhaps the best example of this latter mechanism involves the PPAR $\gamma$ coactivator-1 (PGC-1) family of transcriptional coactivators. PGC-1 coactivators serve as inducible NR "boosters" to equip the organism to meet the energy demands of diverse physiologic and dietary conditions. This Review will focus on the role of this interesting coactivator family in the control of organ-specific biologic responses to the physiologic and pathophysiologic milieu. Emphasis will be given to tissue-specific regulatory features relevant to heart failure and diabetes.

\section{The PGC-1 family: inducible transcriptional coactivators orchestrating control of cellular energy metabolism}

The transcriptional coactivator PGC- $1 \alpha$ was identified through its functional interaction with the nuclear receptor PPAR $\gamma$ in brown adipose tissue (BAT), a mitochondria-rich tissue specialized for thermogenesis (1). Thereafter, 2 related coactivators, PGC-1 $\beta$ (also termed PERC) and PGC-1-related coactivator (PRC), were discovered (Figure 1) (2-4). PGC- $1 \alpha$ and PGC-1 $\beta$ are preferentially expressed in tissues with high oxidative capacity, such as heart, slow-twitch skeletal muscle, and BAT, where they serve critical roles in the regulation of mitochondrial functional capacity and cellular energy metabolism $(1,3,5-7)$. Less is known about the expression patterns and biologic roles of $\operatorname{PRC}(2,8)$.

Nonstandard abbreviations used: AMPK, AMP-activated protein kinase; BAT, brown adipose tissue; CaMK, calcium/calmodulin-dependent protein kinase; ERR, estrogen-related receptor; FOXO1, forkhead box O1; GLUT4, glucose transporter 4; HNF-4, hepatic nuclear factor-4; MEF-2, myocyte enhancer factor-2; NR, nuclear receptor; NRF, nuclear respiratory factor; PGC-1, PPAR $\gamma$ coactivator-1; PRC, PGC-1-related coactivator; RNAi, RNA interference.

Conflict of interest: The authors have declared that no conflict of interest exists. Citation for this article: J. Clin. Invest. 116:615-622 (2006). doi:10.1172/JCI27794.
PGC-1 coactivator docking to specific transcription factors provides a platform for the recruitment of regulatory protein complexes that exert powerful effects on gene transcription (Figure 1). The amino-terminal region of PGC-1 coactivators interacts with proteins containing histone acetyltransferase (HAT) activity, including CREB-binding protein/p300 and steroid receptor coactivator-1 (SRC-1) (9). The HAT activity of this complex remodels histones within chromatin, increasing access of the transcriptional machinery to target genes. A second activating complex, the thyroid hormone receptor-associated protein/vitamin D receptorinteracting protein (TRAP/DRIP, or Mediator) complex, docks on the carboxy terminus of PGC-1 $\alpha$ (10). In addition, PGC- $1 \alpha$ contains several domains within the carboxy-terminal region that couple pre-mRNA splicing with transcription (11).

Earlier studies involving forced overexpression of PGC- $1 \alpha$ or PGC-1 $\beta$ in mammalian cells in culture demonstrated that these coactivators are sufficient to activate gene regulatory programs that drive increased capacity for cellular energy production (3, $5,7,12)$. PGC-1 coactivators effect biologic responses that equip the cell to meet the energy demands of a changing environment, including augmentation of mitochondrial biogenesis, cellular respiration rates, and energy substrate uptake and utilization. The PGC-1 coactivators exert these pleiotropic effects by directly coactivating a specific array of NR and non-NR transcription factors involved in the control of cellular metabolism. Following its discovery as a PPAR $\gamma$ coactivator, Wu et al. demonstrated that PGC- $1 \alpha$ coactivates nuclear respiratory factor- 1 (NRF-1) and -2 (NRF-2) (5). NRFs regulate expression of mitochondrial transcription factor A (Tfam), a nuclear-encoded transcription factor essential for replication, maintenance, and transcription of mitochondrial DNA (13-15). NRF-1 and NRF-2 also control the expression of nuclear genes encoding respiratory chain subunits and other proteins required for mitochondrial function $(16,17)$. These discoveries provided mechanistic insight into how PGC- $1 \alpha$ activates the broad program of mitochondrial biogenesis and revealed that PGC-1 coactivators were capable of interacting with both NR and non-NR transcription factors.

Although PPAR $\gamma$, NRF-1, and NRF-2 are key targets of PGC- $1 \alpha-$ mediated coactivation, the diverse effects of this coactivator could not be explained by these interactions alone. Multiple PGC- $1 \alpha$ partners have now been identified, indicating that this coactivator serves as a pleiotropic regulator of multiple pathways involved in cellular energy metabolism within and outside of the mitochondrion (Figure 2) $(18,19)$. Since the identification of PPAR $\gamma$ as a 
A

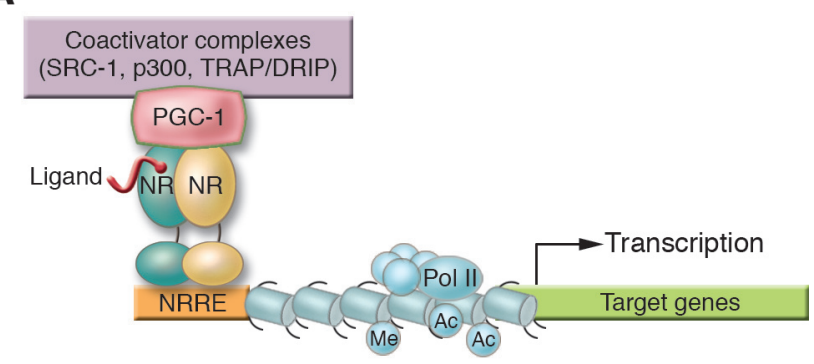

B

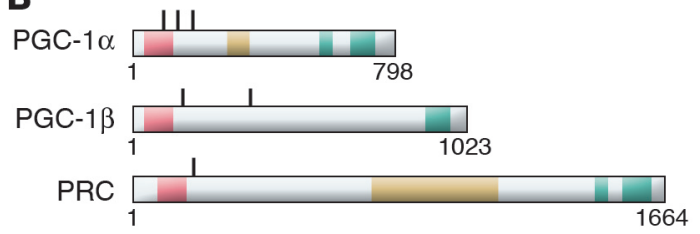

C
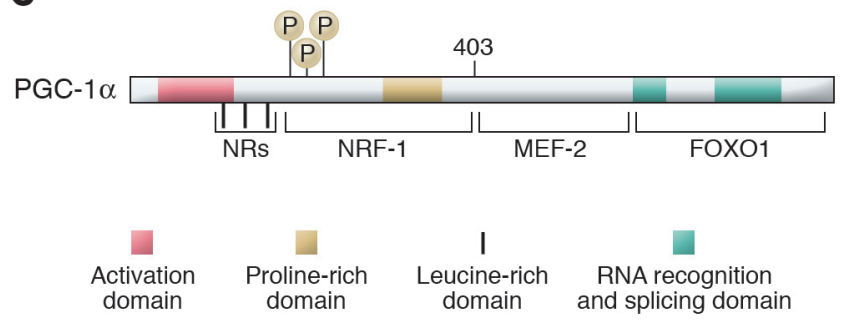

PGC-1 $\alpha$ transcription factor target, a variety of additional PGC-1 target NRs have been identified. This list includes PPAR $\alpha$ (20), PPAR $\beta$ (21), thyroid hormone receptor (1), retinoid receptors (1), glucocorticoid receptor (22), estrogen receptor $(1,22,23)$, farnesyl X receptor (FXR) (24), pregnane X receptor (PXR) (25), hepatic nuclear factor-4 (HNF-4) (26), liver X receptor (LXR) (27), and the estrogen-related receptors (ERRs) $(28,29)$. In addition, several non-NR PGC-1 partners have been identified, including myocyte enhancer factor-2 (MEF-2) (30), forkhead box O1 (FOXO1) (31), SREBP1 (27), and Sry-related HMG box-9 (Sox9) (32). Through these transcription factor partners, PGC-1 exerts strong effects on many aspects of mitochondrial energy metabolism. For example, PGC-1 $\alpha$ coactivates PPAR $\alpha$, a key regulator of genes involved in mitochondrial fatty acid oxidation (20). The PGC-1 target ERR $\alpha$ is an important regulator of mitochondrial energy transduction pathways including fatty acid oxidation and oxidative phosphorylation. In addition, ERR $\alpha$ is capable of cooperating with or directly activating the expression of NRF-1, NRF-2, and PPAR $\alpha$, defining an ERR "cross-regulatory circuit" that theoretically serves as an internal "amplifier" for the PGC- $1 \alpha$ cascade (Figure 2). Finally, several of the PGC-1 coactivation targets regulate pathways outside of the mitochondrion - such as HNF-4 and FOXO1 (gluconeogenesis), MEF-2 (glucose transport), SREBP1 (lipogenesis), and Sox9 (chondrogenesis). In addition to serving a booster function, there is evidence that PGC-1 coactivators also confer target gene specificity. For example, selective activation of PPAR $\gamma$ target genes encoding aP2, uncoupling protein-1, and glycerol kinase is dictated, in part, by the PGC- $1 \alpha /$ PPAR $\gamma$ interaction on these promoters $(1,33)$. The mechanisms involved in the selection of specific targets by PGC- $1 \alpha$ among tissues in a given physiologic context are an important area of investigation.

\section{Figure 1}

The PGC-1 coactivator family: inducible boosters of gene transcription. (A) The schematic uses generic NRs as an example of how inducible PGC-1 coactivators dock to transcription factor targets and recruit protein complexes that activate transcription via either enzymatic modification of chromatin, such as histone acetylation (e.g., by steroid receptor coactivator-1 [SRC-1] or p300), or direct interaction with the transcription initiation machinery (e.g., the thyroid hormone receptor-associated protein/vitamin D receptor-interacting protein [TRAP/DRIP] coactivator complex). The NR binds cognate NR response elements (NRREs) within the promoter region of the target gene. Specific histone modifications, including acetylation (Ac) and methylation (Me), are shown, as is the RNA polymerase II (Pol II) complex. (B) The schematic depicts the relative length and shared domains of the 3 members of the PGC-1 coactivator family. The nature of the domains is indicated in the key. (C) A schematic of the PGC-1 $\alpha$ molecule is shown to denote several key functional domains involved in the interaction with specific target transcription factors including NRs, nuclear respiratory factor-1 (NRF-1), MEF-2, and FOXO1. MAPK phosphorylation (P) sites are also shown.

The discovery of PGC- $1 \alpha$ as a cold-inducible coactivator prompted studies to determine whether its expression is regulated by developmental, physiologic, and dietary cues. One of the first clues that PGC- $1 \alpha$ and PGC- $1 \beta$ serve diverse functions in multiple organ systems was the observation that they are expressed in broad, but tissue-enriched, patterns. PGC- $1 \alpha$ and PGC- $1 \beta$ are highly expressed in mitochondria-enriched tissues with high energy demands, including BAT, heart, and slow-twitch skeletal muscle $(1,3,4)$. PGC-1 $\alpha$ is also enriched in brain and kidney. PGC-1 $\alpha$ expression is induced in the heart after birth in parallel with a postnatal burst of mitochondrial biogenesis and a shift toward reliance on mitochondrial fatty acid oxidation as the major source of ATP production (12). The PGC1A gene is highly inducible in response to physiologic conditions that demand increased mitochondrial energy production. For example, PGC- $1 \alpha$ expression is stimulated by exercise in skeletal muscle (34-38) and by fasting in the heart and liver $(12,26)$. Interestingly, PGC- $1 \beta$ expression is also induced by fasting, but not cold exposure, indicating that factor-specific upstream regulatory circuits exist $(3,39)$.

As would be predicted by its inducibility, the expression and activity of PGC- $1 \alpha$ are linked to a variety of upstream cellular signaling pathways (Figure 2). In BAT and liver (and likely other tissues), the $\beta$-adrenergic/cAMP pathway activates PGC1A gene transcription (1). Calcineurin A and calcium/calmodulin-dependent protein kinase (CaMK) activate PGC-1 $\alpha$ expression in striated muscle (40-42). The AMP-activated protein kinase (AMPK) has also been implicated in the control of muscle PGC-1 $\alpha$ expression (43). p38 MAPK has been shown to activate PGC-1 $\alpha$ by releasing p160-mediated repression and by increasing PGC- $1 \alpha$ protein stability (44-46). More recently, NO was shown to activate mitochondrial biogenesis coincident with increased PGC- $1 \alpha$ expression in a variety of cell types, including adipocytes and HeLa cells (47). Given the known role of NO as a vasodilator, it is tempting to speculate that this key upstream regulatory pathway coordinately regulates downstream events including an increase in the capacity to utilize oxygen in mitochondria. In addition to phosphorylation, other posttranslational modifications, including acetylation, arginine methylation, and interaction with repressor proteins (e.g., p160), modulate PGC- $1 \alpha$ activity $(45,48,49)$.

The transcriptional regulatory factors that link upstream signaling pathways to PGC1A gene expression are being delineated. 
The calcineurin A-mediated activation of the PGC1A promoter is dependent on MEF-2 response elements, whereas CaMK-mediated regulation requires CREB-binding sites (40-42). The transcription factor CREB activates $P G C 1 A$ gene transcription in hepatocytes, implicating this factor in the PGC- $1 \alpha$-mediated control of gluconeogenesis (50). The forkhead transcription factor FOXO1 activates the human $P G C 1 A$ promoter in a hepatoma cell line (51), an effect that is suppressed by protein kinase B/Akt signaling, suggesting a mechanism for negative regulation of PGC-1 $\alpha$ expression by insulin in the liver.

Given that PGC-1 $\alpha$ integrates regulatory input from a variety of upstream regulatory pathways among multiple tissues, in vivo studies have been necessary to define the bona fide biologic functions downstream of this powerful transcriptional coactivator. In vivo studies have been empowered by the development of generalized and conditional transgenic PGC- $1 \alpha$ gain-of-function mouse models. In addition, 2 independent generalized PGC- $1 \alpha$-deficient mouse lines ( $\mathrm{Pgcla}^{-/-}$mice) have been generated using gene targeting strategies $(52,53)$. Both lines are viable and exhibit multisystem energy metabolic abnormalities that were unveiled by physiologic or nutritional stressors.

\section{PGC-1 signaling and the heart}

The extraordinary energy demands of the heart are met by a highcapacity mitochondrial system. Accordingly, it is not surprising that PGC- $1 \alpha$ and PGC- $1 \beta$ are highly expressed in this organ $(1,3$, 54). Although the exact roles of PGC-1 $\beta$ and PRC in heart have not been defined, several lines of evidence indicate that PGC- $1 \alpha$ controls cardiac energy metabolic pathways during development and in response to physiologic stressors. First, cardiac PGC-1 $\alpha$ expression is induced after birth as the heart turns toward mitochondrial fatty acid oxidation as the chief energy source (12). PGC-1 $\alpha$ expression is also activated in the heart by fasting (12), a physiologic stimulus that markedly increases the reliance of the heart on mitochondrial fat oxidation for ATP production. Second, PGC- $1 \alpha$ coactivates PPAR $\alpha$ and ERR $\alpha$, NRs that control genes involved in cardiac fatty acid oxidation and mitochondrial respiratory function (55). Third, forced overexpression of PGC- $1 \alpha$ in cardiac myocytes in culture activates mitochondrial biogenesis (12). Interestingly, in contrast to the BAT, where PGC- $1 \alpha$ drives uncoupled respiration, PGC- $1 \alpha$ overexpression drives coupled respiration in the cardiac myocyte (12).

Gain-of-function studies in transgenic mice have demonstrated potential biologic functions of PGC- $1 \alpha$ in the heart in vivo. Two

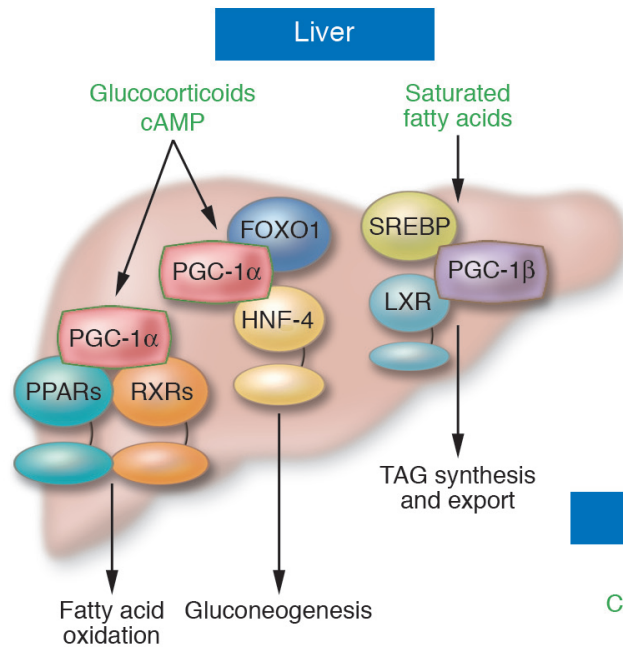

Striated muscle

p38 MAPK Calcineurin A/CaMKII AMPK
Brown adipose tissue

$\beta_{3}$-adrenergic/cAMP pathway

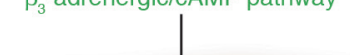

$\downarrow$

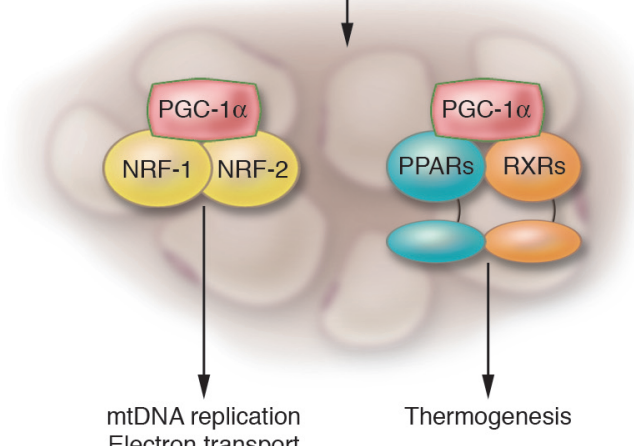

Electron transport OXPHOS

\section{Figure 2}

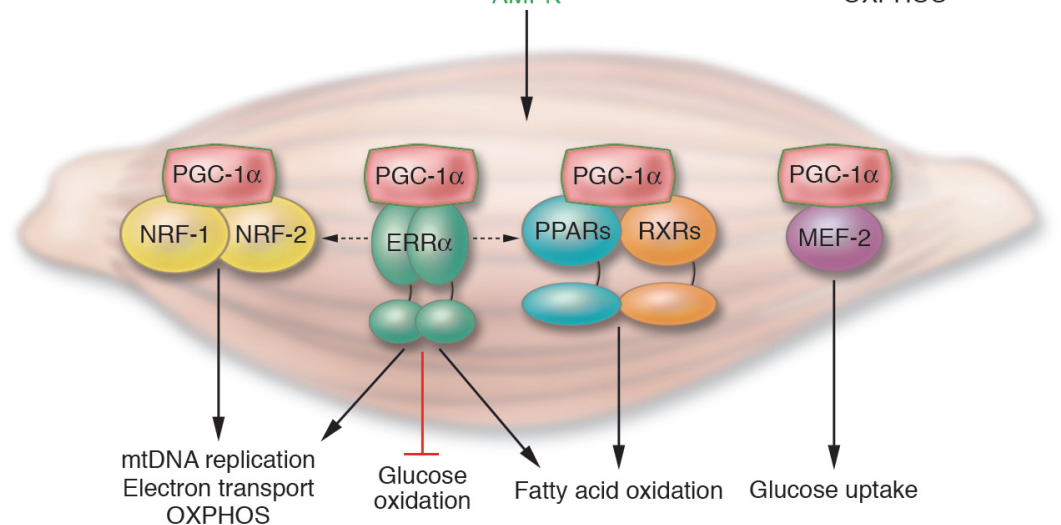

The PGC-1 gene regulatory cascade. The schematic indicates the upstream signaling events and downstream gene regulatory actions of the inducible PGC- 1 coactivators, using PGC- $1 \alpha$ as the representative factor. The interaction of PGC- $1 \alpha$ with its cognate transcription factor targets is shown linked to specific organ systems. For example, PGC-1 $\alpha$ coactivates members of the PPAR nuclear receptor transcription factor family, to activate the expression of genes involved in mitochondrial fatty acid oxidation. The signaling pathways shown at the top of each organ system transduce extracellular physiologic and nutritional stimuli to the expression and/or activity of PGC-1 $\alpha$. LXR, liver X receptor; TAG, triacylglycerol; RXR, retinoid $\mathrm{X}$ receptor; mtDNA, mitochondrial DNA; OXPHOS, oxidative phosphorylation. 
transgenic mouse models with cardiac-restricted overexpression of PGC- $1 \alpha$ have been developed. In the first model, mice with constitutive, postnatal cardiac overexpression of PGC- $1 \alpha$ under control of the cardiac $\alpha$-myosin heavy chain promoter (MHCPGC- $1 \alpha$ mice) were generated (12). The hearts of MHC-PGC-1 $\alpha$ mice exhibit marked activation of cardiac myocyte mitochondrial biogenesis, leading, ultimately, to death from heart failure (12). Subsequently, a bitransgenic, tissue-specific, tetracycline-inducible PGC- $1 \alpha$ mouse was established (the cs-tet-on PGC-1 $\alpha$ mouse) (56). Studies of cs-tet-on PGC- $1 \alpha$ mice revealed developmental stage-specific effects of PGC- $1 \alpha$. Overexpression of PGC- $1 \alpha$ in neonatal heart triggers dramatic expansion of mitochondria within the cardiac myocytes. In contrast, acute cardiac overexpression of PGC- $1 \alpha$ in adult mice results in a modest mitochondrial biogenic response and, after several weeks, cardiomyopathy associated with mitochondrial ultrastructural abnormalities. The basis for cardiomyopathy following PGC-1 $\alpha$ activation is unknown but likely involves dysregulated mitochondrial metabolism.

Recent loss-of-function studies further support a role for PGC- $1 \alpha$ in the control of myocardial metabolism and function. Interestingly, the severity of the cardiac phenotype varies between the 2 independently generated lines of PGC- $1 \beta$-deficient mice. Both lines demonstrate that PGC-1 $\alpha$ is not essential for the fundamental process of mitochondrial biogenesis in the heart; myocardial mitochondrial volume density is not significantly altered in either Pgc1 $a^{-/-}$line $(53,57)$. The PGC-1 $\alpha$-deficient mice produced by the Spiegelman group exhibit moderate, age-related base-line cardiac dysfunction as determined by echocardiography (57). Hearts isolated from this line of mice also exhibit a modest impairment in maintenance of ATP and phosphocreatine homeostasis in response to the $\beta$-adrenergic agonist dobutamine as determined by NMR spectroscopy. These metabolic abnormalities are associated with diminished expression of genes involved in mitochondrial fatty acid oxidation, the TCA cycle, and oxidative phosphorylation. In contrast, the PGC-1 $\alpha$-deficient mice produced by Leone et al. do not exhibit overt dysfunction by echocardiography under basal conditions; however, this second line of $\mathrm{Pgcla}^{-/-}$mice exhibit a blunted heart rate response to exercise and $\beta$-adrenergic stimulation (53). The mechanistic basis for the dissimilarities between the 2 lines of $\mathrm{Pgcla}^{-/-}$mice is unclear. It is possible that epigenetic effects due to differences in strain background or gene targeting strategies account for the divergent phenotypes.

Several lines of recent evidence implicate PGC-1 $\alpha$ in the pathogenesis of heart failure. First, in mouse models of chronic pressure overload such as occurs in long-standing hypertension, PGC- $1 \alpha$ levels are downregulated along with the expression of PPAR $\alpha$ target genes involved in mitochondrial fatty acid oxidation (58-60). Second, transcriptional profiling revealed downregulation of PGC- $1 \alpha$ gene expression in the failing hearts of transgenic mice in which cyclin $\mathrm{T} / \mathrm{Cdk} 9$, an RNA polymerase kinase, is overexpressed in the heart $(61,62)$. Forced expression of PGC-1 $\alpha$ in the mutant cardiac myocytes prevented the apoptotic phenotype (62). These results are interesting given that mitochondrial respiratory function and high-energy phosphate stores are known to be reduced in end-stage heart failure (63). Lastly, chronic overexpression of PGC-1 $\alpha$ in heart leads to a cardiomyopathic phenotype $(12,56)$. Thus, emerging evidence links altered PGC-1 $\alpha$ activity to cardiac dysfunction. However, whether PGC- $1 \alpha$ plays an etiologic role in the pathogenesis of heart failure remains to be determined.

\section{PGC-1 signaling and skeletal muscle}

The expression of PGC- $1 \alpha$ is enriched in skeletal muscle, particularly in oxidative fiber types. Studies in rodent models indicate that both short-term exercise and endurance training activate PGC-1 $\alpha$ expression in skeletal muscle $(34,35,64,65)$. Subsequent studies in humans have further demonstrated remarkable inducibility of PGC- $1 \alpha$ in response to acute bouts of exercise or endurance training $(38,66)$ and have indicated that PGC- $1 \alpha$ levels are increased mainly in type IIa fibers after endurance training (66). Although the upstream signaling events involved in the activation of PGC-1 $\alpha$ expression in exercising muscle are not well defined, several pathways have been implicated. The calcineurin A and CaMK pathways are linked to muscle $P G C 1 A$ gene transcription through MEF-2 factors (40-42). Recently, the p38 MAPK and AMPK pathways have also been implicated in the control of PGC-1 $\alpha$ expression in skeletal muscle after exercise training $(43,67)$.

The biologic roles of PGC- $1 \alpha$ in skeletal muscle have been elucidated largely through murine gain-of-function and loss-of-function studies. Lin et al. developed mice with skeletal muscle-specific overexpression of PGC- $1 \alpha$ driven by the muscle creatine kinase (MCK) promoter (68). Forced expression of PGC-1 $\alpha$ at high physiologic levels in the MCK transgenic mice resulted in an increased proportion of oxidative or type I muscle fibers coincident with an increase in the expression of mitochondrial markers. This fiber type transition was also manifest by red muscle coloration, increased expression of contractile protein characteristic of type I fibers, and resistance to electrically stimulated fatigue (68). These results indicated that PGC- $1 \alpha$ is sufficient to drive the slowtwitch skeletal muscle program. Complementary loss-of-function studies demonstrated that $\mathrm{Pgcla}^{-/-}$mice exhibit diminished mitochondrial number and respiratory capacity in slow-twitch skeletal muscle (53). In contrast, measures of mitochondrial function and density are normal in fast-twitch fibers of the PGC- $1 \alpha$-deficient mice. However, exercise capacity, as measured on a motorized treadmill, and fatigue resistance index in electrically stimulated muscle, are significantly reduced in the $P g c 1 a^{-/-}$mice (53). Taken together, these results strongly implicate PGC- $1 \alpha$ signaling as a key mediator of the energy metabolic and structural adaptation of muscle to exercise.

Recent evidence also implicates PGC- $1 \alpha$ in the regulation of muscle glucose metabolism. The first clue came from studies demonstrating that PGC- $1 \alpha$ robustly activates expression of glucose transporter 4 (GLUT4) in skeletal muscle cells in culture by coactivating MEF-2c (30). More recently, PGC-1 $\alpha$ was found to repress glucose oxidation in muscle cell lines by activating the expression of the gene encoding pyruvate dehydrogenase kinase 4 via its NR partner, ERR $\alpha$ (69). These latter results suggest that PGC- $1 \alpha$ controls muscle fuel selection by increasing fatty acid oxidation while temporarily shutting down glucose oxidation - a gene regulatory equivalent of the fatty acid-glucose cycle. Inhibition of glucose oxidation combined with increased muscle glucose uptake could serve to replenish muscle glycogen stores to prepare for the next bout of exercise.

\section{PGC-1 signaling and hepatic function}

Under normal, ad libitum-fed conditions, the expression of PGC- $1 \alpha$ and PGC- $1 \beta$ is relatively low in liver compared with other tissues that rely on aerobic metabolism for ATP production $(1,39)$. However, hepatic $P G C 1 A$ and $P G C 1 B$ gene expression is robustly induced by fasting $(39,50,70)$. During short-term star- 
vation, the fatty acid oxidation pathway is a key source of substrates for ATP production, ketogenesis, and gluconeogenesis. PGC- $1 \alpha$ and PGC- $1 \beta$ activate expression of PPAR $\alpha$ target genes involved in hepatic fatty acid oxidation $(39,70,71)$, and acute RNA interference-mediated (RNAi-mediated) PGC- $1 \alpha$ knock down leads to profound downregulation of fatty acid oxidation gene expression (71). Rates of fatty acid oxidation are also diminished in hepatocytes isolated from PGC-1 $\alpha$-deficient mice (53). The reduction in fatty acid oxidative capacity likely contributes to the fasting-induced hepatic steatotic phenotype observed in one of the mutant lines (53). Surprisingly, the expression of PPAR $\alpha$ target genes involved in $\beta$-oxidation is not reduced in generalized Pgcla $a^{-/}$mice $(52,53)$. However, hepatocytes isolated from PGC-1 $\alpha$-deficient mice exhibit diminished mitochondrial respiration rates (53), providing one mechanistic explanation for the reduced capacity for hepatic fatty acid oxidation.

During periods of fasting, the liver catabolizes fatty acids to produce 3-carbon substrates for gluconeogenesis, the most important source of de novo glucose production in higher organisms. $P G C 1 A$ gene expression is activated in liver by fasting $(50,70)$ and glucocorticoids (72), and it is now recognized that it regulates hepatic gluconeogenesis $(31,50,70,71)$. Ectopic expression of PGC- $1 \alpha$ coactivates HNF- $4 \alpha$ and FOXO1 to drive expression of genes involved in gluconeogenesis (31), whereas PGC-1 $\beta$ does not activate this pathway (39). The nutrient sensor SIRT1, which is linked to longevity in several animal models, also exerts critical posttranslational regulatory control of gluconeogenic gene expression via deacetylation of PGC-1 $\alpha$ (48). Liver-specific PGC1A gene deletion studies (73) and acute RNAi-based PGC-1 $\alpha$ hepatic knockdown strategies (71) in mice lead to diminished expression of genes encoding gluconeogenic enzymes. After short-term starvation, one of the lines of generalized $\mathrm{Pgcla}^{-/-}$mice becomes hypoglycemic (52), consistent with a defect in the hepatic gluconeogenic response. However, the basal expression of key gluconeogenic genes such as PEPCK is either normal (53) or increased (52) in the PGC-1 $\alpha$-deficient lines. Accordingly, it is not clear whether the major regulatory effect of PGC-1 $\alpha$ on this pathway occurs via direct transcriptional control of gluconeogenic genes or secondarily to reductions in flux through fatty acid oxidation or the TCA cycle, which are coupled to, and required for, gluconeogenesis.
A striking example of gene target selectivity between PGC-1 $\alpha$ and PGC-1 $\beta$ was recently unveiled in liver. Administration of a diet enriched in saturated or trans-fatty acids resulted in acute induction of hepatic PGC- $1 \beta$ expression without altering PGC- $1 \alpha$ expression (27). PGC-1 $\beta$ overexpression in liver stimulated hepatic triglyceride production and secretion, resulting in circulating hypertriglyceridemia and hypercholesterolemia. Conversely, it was previously shown that activation of PGC-1 $\alpha$ in liver diminished triglyceride production and secretion (24). The mechanistic explanation for these surprising results came from gene regulatory studies demonstrating that PGC- $1 \beta$, but not PGC- $1 \alpha$, activates the expression of genes involved in lipogenesis and triglyceride secretion via direct coactivation of SREBP1c (27), a transcription factor that plays a critical role in the control of lipogenesis. The region of PGC- $1 \beta$ that mediates the protein-protein interaction with SREBP is not conserved among PGC-1 family members; this provides an explanation for the observed isoform selectivity. This work provides a potential mechanistic basis for the known association between diets high in saturated or trans-fat and hyperlipidemia.

In sum, evidence is mounting that induction of PGC- $1 \alpha$ and PGC- $1 \beta$ expression in liver is a critical regulatory event leading to the activation of energy metabolic pathways that serve to increase ATP production and exert homeostatic control, especially in the context of fasting. Given the importance of hepatic lipid metabolic derangements in common diseases such as nonalcoholic steatotic hepatitis and alcoholic liver disease, as well as the link between liver insulin resistance and diabetes, the PGC-1 regulatory circuits represent potential new therapeutic targets for hepatic disease states.

\section{Paradoxical roles for PGC-1 $\alpha$ in insulin resistance and diabetes mellitus}

Recent studies in animal models and in humans link altered PGC-1 $\alpha$ signaling to glucose intolerance, insulin resistance, and diabetes. However, the role of PGC- $1 \alpha$ as a protective factor versus mediator of disease progression is unclear, particularly given that its predicted effects on insulin sensitivity and glucose tolerance vary across tissues (Figure 3). Associations between PGC-1 $\alpha$ and diabetes have been identified in studies of human genetic variants. A common polymorphism in the coding region of the PGC1A gene (Gly482Ser) and a specific promoter haplotype are associated with

\section{A Type 2 diabetes}

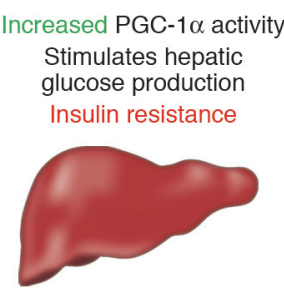

Liver

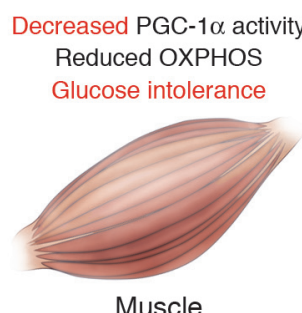

Muscle
Increased PGC- $1 \alpha$ activity Suppresses insulin secretion Insulin deficiency

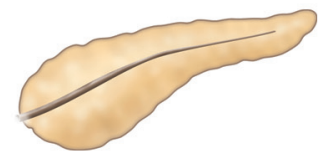

Pancreas

\section{B PGC-1 $\alpha$-deficient mice}

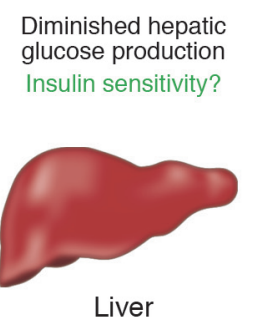

Reduced OXPHOS Insulin resistance?

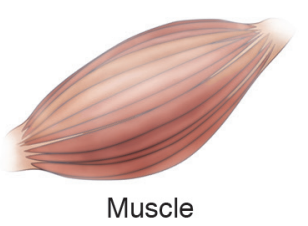

Figure 3

Potential contributions of organ-specific dysregulation of PGC-1 $\alpha$ to the development of insulin resistance and type 2 diabetes. (A) PGC-1 $\alpha$ expression and activity have been shown to be increased in the liver and pancreatic $\beta$ cell in several animal models of diabetes mellitus. Conversely, gene expression profiling indicates that PGC-1 $\alpha$ expression is diminished in skeletal muscle of type 1 and 2 diabetic humans along with reduced expression of genes involved in oxidative phosphorylation (OXPHOS). This tissue-specific pattern of dysregulated PGC-1 $\alpha$ activity is predicted to potentially contribute to systemic insulin resistance, glucose intolerance, and insulin deficiency. (B) The generalized PGC-1 $\alpha-$ deficient mouse is relatively protected against diet-induced insulin resistance and glucose intolerance despite impairments in skeletal muscle OXPHOS capacity. Improved insulin sensitivity may stem from diminished hepatic glucose production, a principal constituent of whole-body glucose homeostasis. However, the relative contribution of individual organ systems to the systemic insulin-sensitive phenotype requires further investigation. 
an increased risk of type 2 diabetes (74-81). However, other studies have not found this association $(82,83)$. Additionally, the target tissues affected and whether these polymorphisms result in increased or decreased PGC- $1 \alpha$ activity remain unclear.

PGC- $1 \alpha$ activity is robustly activated in diabetic liver as it is in the fasted state $(26,31,50,70)$, potentially increasing hepatic glucose production that contributes to circulating hyperglycemia. Moreover, PGC- $1 \alpha$ may promote insulin resistance directly by inducing TRB-3, an inhibitor of Akt signaling, a critical downstream component of the insulin signaling pathway (71). The precise mechanisms involved in the regulatory cross-talk between insulin signaling and PGC- $1 \alpha$ activity in the diabetic state represent an area of active research.

In the pancreatic $\beta$ cell, rates of mitochondrial ATP synthesis are inexorably coupled to insulin secretion by ATP-sensing $\mathrm{K}^{+}$channels, which control insulin release. PGC- $1 \alpha$ is also activated in the $\beta$ cell in several rodent models of obesity and type 2 diabetes (84), and PGC-1 $\alpha$ overexpression suppressed glucose-stimulated membrane depolarization and insulin secretion in cultured islets or insulinoma cell lines (84). Moreover, whereas transplantation of normal islets into mice rendered diabetic by streptozotocin returned blood glucose levels to normal, PGC- $1 \alpha$-overexpressing islets were unable to reverse experimentally induced diabetes. The $\beta$ cell phenotype of the PGC- $1 \alpha$-deficient mice and the effects of other PGC- 1 family members in the $\beta$ cell have not yet been delineated.

In contrast to results in liver and the $\beta$ cell, the bulk of evidence focused on skeletal muscle suggests that PGC-1 $\alpha$ may be protective from the development of insulin resistance. First, as described above, PGC- $1 \alpha$ activates expression of GLUT4 (30). Second, PGC-1 $\beta$ overexpression in skeletal muscle protects mice from high-fat diet-induced obesity and insulin resistance (6). Third, inherited mitochondrial deficiencies are linked with the development of systemic metabolic defects and diabetes (85). Accordingly, PGC- $1 \alpha$, a critical booster of mitochondrial function, is an excellent candidate for preventing insulin resistance secondary to mitochondrial dysfunction. Fourth, a series of recent studies in humans have shown an interesting inverse correlation between muscle PGC- $1 \alpha$ levels and mitochondrial activity with insulin resistance and diabetes (86-88). However, a separate recent study did not find a correlation between muscle mitochondrial derangements and PGC- $1 \alpha$ levels in insulin-resistant humans (89). Given that the published results focused on PGC- $1 \alpha$ levels in insulin-resistant muscle are largely correlative, the role of alterations in PGC- $1 \alpha$ activity as etiologic versus a secondary phenomenon remains unclear.

One approach to unraveling the paradoxical, tissue-specific actions of PGC- $1 \alpha$ on systemic glucose metabolism and insulin sensitivity is through studies of recently developed genetically modified mouse models. Systemic glucose tolerance and insulin sensitivity have been characterized in both PGC-1 $\alpha$-deficient lines. Although some differences exist in the 2 lines, the results indicate that both $\mathrm{Pgcla}^{-/-}$lines are modestly protected against insulin resistance caused by high-fat diet. Interestingly, this insulin-sensitive phenotype occurs despite reduced respiratory capacity of mitochondria in the skeletal muscle of the mice (53). It is possible that reduced hepatic glucose production via the gluconeogenic pathway contributes to the enhanced glucose tolerance of PGC-1 $\alpha$-deficient mice (Figure 3 ). Indeed, as described above, liver-specific overexpression and knockdown strategies indicate that PGC-1 $\alpha$, when activated, drives hepatic glucose production, a potential contributor to the development of obesity-related diabetes. In addition, one of the PGC- $1 \alpha$-deficient lines exhibited increased activity and partial resistance to weight gain, which could contribute to the phenotype (52). Given the mounting evidence of a link between diabetes and PGC-1 $\alpha$ activity, this represents an important focus for future investigation.

\section{PGC-1 coactivators: the future}

A recent explosion of new information indicates that the PGC-1 family of coactivators serves key functions in the dynamic transcriptional control of energy metabolic pathways in a variety of mammalian tissues. The importance of PGC- $1 \alpha$ and PGC- $1 \beta$ as boosters of $\mathrm{NR}$ function has provided exciting new avenues for understanding the fundamental connections between alterations in the external environment and adaptive metabolic responses of striated muscle and liver. Moreover, evidence is mounting that dysregulation of the PGC- $1 \alpha$ regulatory axis contributes to the pathogenesis of common disease states including diabetes and heart failure. However, the role of these powerful and highly inducible coactivators as protectors versus mediators of disease has not been well defined and will require additional translational studies bridging animal models, such as conditional genetically modified mouse models, and humans. Given the versatile and pleiotropic nature of the PGC-1 family regulatory circuit, it is envisioned that, after the elucidation of the precise roles of these coactivators in disease states, specific PGC-1/NR regulatory limbs could prove to be exciting new therapeutic targets.

\section{Acknowledgments}

The authors wish to thank Mary Wingate for expert assistance in preparing this manuscript and Janice Huss for valuable contributions. Part of the work described in this Review was supported by NIH grants R01 DK45416, R01 HL58493, P50 HL077113, and P01 HL57278; the Clinical Nutrition Research Unit Core Center (P30 DK56341); and the Digestive Diseases Research Core Center (P30 DK52574). B.N. Finck receives support from an NIH grant (K01 DK062903).

Address correspondence to: Daniel P. Kelly, Washington University School of Medicine, 660 South Euclid Avenue, Campus Box 8086, St. Louis, Missouri 63110, USA. Phone: (314) 362-8908; Fax: (314) 362-0186; E-mail: dkelly@im.wustl.edu.
1. Puigserver, P., et al. 1998. A cold-inducible coactivator of nuclear receptors linked to adaptive thermogenesis. Cell. 92:829-839.

2. Andersson, U., and Scarpulla, R.C. 2001. PGC-1related coactivator, a novel, serum-inducible coactivator of nuclear respiratory factor-1-dependent transcription in mammalian cells. Mol. Cell. Biol. 21:3738-3749.

3. Lin, J., Puigserver, P., Donovan, J., Tarr, P., and Spiegelman, B.M. 2002. Peroxisome proliferator-acti- vated receptor $\gamma$ coactivator $1 \beta$ (PGC-1 $\beta$ ), a novel PGC-1-related transcription coactivator associated with host cell factor. J. Biol. Chem. 277:1645-1648.

4. Kressler, D., Schreiber, S.N., Knutti, D., and Kralli, A. 2002. The PGC-1-related protein PERC is a selective coactivator of estrogen receptor alpha. J. Biol. Chem. 277:13918-13925.

5. Wu, Z., et al. 1999. Mechanisms controlling mitochondrial biogenesis and respiration through the thermogenic coactivator PGC-1. Cell. 98:115-124.
6. Kamei, Y., et al. 2003. PPAR $\gamma$ coactivator $1 \beta /$ ERR ligand 1 is an ERR protein ligand, whose expression induces a high-energy expenditure and antagonizes obesity. Proc. Natl. Acad. Sci. U. S. A. 100:12378-12383.

7. St-Pierre, J., et al. 2003. Bioenergetic analysis of peroxisome proliferator-activated receptor $\gamma$ coactivators $1 \alpha$ and $1 \beta$ (PGC- $1 \alpha$ and PGC- $1 \beta$ ) in muscle cells. J. Biol. Chem. 278:26597-26603.

8. Savagner, F., et al. 2003. PGC-1-related coactivator 
and targets are upregulated in thyroid oncocytoma. Biochem. Biophys. Res. Commun. 310:779-784.

9. Puigserver, P., et al. 1999. Activation of PPAR $\gamma$ coactivator-1 through transcription factor docking. Science. 286:1368-1371.

10. Wallberg, A.E., Yamamura, S., Malik, S., Spiegelman, B.M., and Roeder, R.G. 2003. Coordination of p300-mediated chromatin remodeling and TRAP/ mediator function through coactivator PGC-1 $\alpha$. Mol. Cell. 12:1137-1149.

11. Monsalve, M., et al. 2000. Direct coupling of transcription and mRNA processing through the thermogenic coactivator PGC-1. Mol. Cell. 6:307-316.

12. Lehman, J.J., et al. 2000. PPAR $\gamma$ coactivator-1 (PGC-1) promotes cardiac mitochondrial biogenesis. J. Clin. Invest. 106:847-856.

13. Fisher, R.P., Lisowsky, T., Parisi, M.A., and Clayton, D.A. 1992. DNA wrapping and bending by a mitochondrial high mobility group-like transcriptional activator protein. J. Biol. Chem. 267:3358-3367.

14. Garesse, R., and Vallejo, C.G. 2001. Animal mitochondrial biogenesis and function: a regulatory cross-talk between two genomes. Gene. 263:1-16.

15. Larsson, N.-G., et al. 1998. Mitochondrial transcription factor A is necessary for mtDNA maintenance and embryogenesis in mice. Nat. Genet. 18:231-236.

16. Scarpulla, R.C. 2002. Nuclear activators and coactivators in mammalian mitochondrial biogenesis. Biochim. Biophys. Acta. 1576:1-14.

17. Virbasius, C.A., Virbasius, J.V., and Scarpulla, R.C. 1993. NRF-1, an activator involved in nuclear-mitochondrial interactions, utilizes a new DNA-binding domain conserved in a family of developmental regulators. Genes Dev. 7:2431-2445.

18. Knutti, D., and Kralli, A. 2001. PGC-1, a versatile coactivator. Trends Endocrinol. Metab. 12:360-365.

19. Puigserver, P., and Spiegelman, B.M. 2003. Peroxisome proliferator-activated receptor- $\gamma$ coactivator $1 \alpha$ (PGC- $1 \alpha)$ : transcriptional coactivator and metabolic regulator. Endocr. Rev. 24:78-90.

20. Vega, R.B., Huss, J.M., and Kelly, D.P. 2000. The coactivator PGC-1 cooperates with peroxisome proliferator-activated receptor $\gamma$ in transcriptional control of nuclear genes encoding mitochondrial fatty acid oxidation enzymes. Mol. Cell. Biol. 20:1868-1876.

21. Wang, Y.-X., et al. 2003. Peroxisome-proliferatoractivated receptor $\gamma$ activates fat metabolism to prevent obesity. Cell. 113:159-170.

22. Knutti, D., Kaul, A., and Kralli, A. 2000. A tissuespecific coactivator of steroid receptors. Mol. Cell. Biol. 20:2411-2422.

23. Tcherepanova, I., Puigserver, P., Norris, J.D., Speigelman, B.M., and McDonnell, D.P. 2000. Modulation of estrogen receptor- $\alpha$ transcriptional activity by the coactivator PGC-1. J. Biol. Chem. 275:16302-16308.

24. Zhang, Y., Castellani, L.W., Sinal, C.J., Gonzalez, F.J., and Edwards, P.A. 2004. Peroxisome proliferator-activated receptor- $\gamma$ coactivator $1 \alpha(\mathrm{PGC}-1 \alpha)$ regulates triglyceride metabolism by activation of the nuclear receptor FXR. Genes Dev. 18:157-169.

25. Bhalla, S., Ozalp, C., Fang, S., Xiang, L., and Kemper, J.K. 2004. Ligand-activated pregnane X receptor interferes with HNF-4 signaling by targeting a common coactivator PGC-1 $\alpha$. Functional implications in hepatic cholesterol and glucose metabolism. J. Biol. Chem. 279:45139-45147.

26. Rhee, J., et al. 2003. Regulation of hepatic fasting response by PPAR $\gamma$ coactivator- $1 \alpha$ (PGC- 1 ): requirement for hepatocyte nuclear factor $4 \alpha$ in gluconeogenesis. Proc. Natl. Acad. Sci. U. S. A. 100:4012-4017.

27. Lin, J., et al. 2005. Hyperlipidemic effects of dietary saturated fats mediated through PGC- $1 \beta$ coactivation of SREBP. Cell. 120:261-273.

28. Huss, J.M., Kopp, R.P., and Kelly, D.P. 2002. PGC$1 \alpha$ coactivates the cardiac-enriched nuclear recep- tors estrogen-related receptor- $\alpha$ and $-\gamma$. J. Biol. Chem. 277:40265-40274.

29. Schreiber, S.N., Knutti, D., Brogli, K., Uhlmann, T., and Kralli, A. 2003. The transcriptional coactivator PGC-1 regulates the expression and activity of the orphan nuclear receptor estrogen-related receptor $\alpha$ (ERR $\alpha)$. J. Biol. Chem. 278:9013-9018.

30. Michael, L.F., et al. 2001. Restoration of insulin-sensitive glucose transporter (GLUT4) gene expression in muscle cells by the transcriptional coactivator PGC-1. Proc. Natl. Acad. Sci. U. S. A. 98:3820-3825.

31. Puigserver, P., et al. 2003. Insulin-regulated hepatic gluconeogenesis through FOXO1-PGC-1 $\alpha$ interaction. Nature. 423:550-555.

32. Kawakami, Y., et al. 2005. Transcriptional coactivator PGC- $1 \alpha$ regulates chondrogenesis via association with Sox9. Proc. Natl. Acad. Sci. U. S. A. 102:2414-2419.

33. Guan, H.-P., Ishizuka, T., Chui, P.C., Lehrke, M., and Lazar, M.A. 2005. Corepressors selectively control the transcriptional activity of PPAR $\gamma$ in adipocytes. Genes Dev. 19:453-461.

34. Goto, M., et al. 2000. cDNA cloning and mRNA analysis of PGC-1 in epitrochlearis muscle in swimming-exercised rats. Biochem. Biophys. Res. Commun. 274:350-354

35. Baar, K., et al. 2002. Adaptations of skeletal muscle to exercise: rapid increase in the transcriptional coactivator PGC-1. FASEB J. 16:1879-1886.

36. Terada, S., et al. 2002. Effects of low-intensity prolonged exercise on PGC-1 mRNA expression in rat epitrochlearis muscle. Biochem. Biophys. Res. Commun. 296:350-354.

37. Terada, S., and Tabata, I. 2003. Effects of acute bouts of running and swimming exercise on PGC- $1 \alpha$ protein expression in rat epitrochlearis and soleus muscle. Am. J. Physiol. Endocrinol. Metab. 286:E208-E216.

38. Pilegaard, H., Saltin, B., and Neufer, P.D. 2003 Exercise induces transient transcriptional activation of the PGC-1 $\alpha$ gene in human skeletal muscle. J. Physiol. 546:851-858.

39. Lin, J., et al. 2003. PGC-1beta in the regulation of hepatic glucose and energy metabolism. J. Biol. Chem. 278:30843-30848.

40. Czubryt, M.P., McAnally, J., Fishman, G.I., and Olson, E.N. 2003. Regulation of peroxisome proliferator-activated receptor $\gamma$ coactivator $1 \alpha($ PGC- $1 \alpha)$ and mitochondrial function by MEF2 and HDAC5. Proc. Natl. Acad. Sci. U. S. A. 100:1711-1716.

41. Handschin, C., Rhee, J., Lin, J., Tam, P.T., and Spiegelman, B.M. 2003. An autoregulatory loop controls peroxisome proliferator-activated receptor $\gamma$ coactivator $1 \alpha$ expression in muscle. Proc. Natl. Acad. Sci. U. S. A. 100:7111-7116.

42. Schaeffer, P.J., et al. 2004. Calcineurin and calcium/ calmodulin-dependent protein kinase activate distinct metabolic gene regulatory programs in cardiac muscle. J. Biol. Chem. 279:39593-39603.

43. Zong, H., et al. 2002. AMP kinase is required for mitochondrial biogenesis in skeletal muscle in response to chronic energy deprivation. Proc. Natl. Acad. Sci. U. S. A. 99:15983-15987.

44. Puigserver, P., et al. 2001. Cytokine stimulation of energy expenditure through $\mathrm{p} 38$ MAP kinase activation of PPAR $\gamma$ coactivator-1. Mol. Cell. 8:971-982.

45. Knutti, D., Kressler, D., and Kralli, A. 2001. Regulation of the transcriptional coactivator PGC-1 via MAPK-sensitive interaction with a corepressor. Proc. Natl. Acad. Sci. U. S. A. 98:9713-9718.

46. Fan, M., et al. 2004. Suppression of mitochondrial respiration through recruitment of $160 \mathrm{myb}$ binding protein to PGC-1alpha: modulation by p38 MAPK. Genes Dev. 18:278-289.

47. Nisoli, E., et al. 2003. Mitochondrial biogenesis in mammals: the role of endogenous nitric oxide. Science. 299:896-899.

48. Rodgers, J.T., et al. 2005. Nutrient control of glucose homeostasis through a complex of PGC-1 $1 \alpha$ and SIRT1. Nature. 434:113-118.

49. Teyssier, C., Ma, H., Emter, R., Kralli, A., and Stallcup, M.R. 2005. Activation of nuclear receptor coactivator PGC- $1 \alpha$ by arginine methylation. Genes Dev. 19:1466-1473.

50. Herzig, S., et al. 2001. CREB regulates hepatic gluconeogenesis through the coactivator PGC-1. Nature. 413:179-183.

51. Daitoku, H., Yamagata, K., Matsuzaki, H., Hatta, M., and Fukamizu, A. 2003. Regulation of PGC-1 promoter activity by protein kinase $\mathrm{B}$ and the forkhead transcription factor FKHR. Diabetes. 52:642-649.

52. Lin, J., et al. 2004. Defects in adaptive energy metabolism with CNS-linked hyperactivity in PGC-1 $\alpha$ null mice. Cell. 119:121-135.

53. Leone, T.C., et al. 2005. PGC- $1 \alpha$ deficient mice exhibit multi-system energy metabolic derangements: muscle dysfunction, abnormal weight control, and hepatic steatosis. PLoS Biol. 3:672-687.

54. Mootha, V.K., et al. 2003. Integrated analysis of protein composition, tissue diversity, and gene regulation in mouse mitochondria. Cell. 115:629-640.

55. Huss, J.M., and Kelly, D.P. 2004. Nuclear receptor signaling and cardiac energetics. Circ. Res. 95:568-578.

56. Russell, L.K., et al. 2004. Cardiac-specific induction of the transcriptional coactivator peroxisome proliferator-activated receptor $\gamma$ coactivator- $1 \alpha$ promotes mitochondrial biogenesis and reversible cardiomyopathy in a developmental stage-dependent manner. Circ. Res. 94:525-533.

57. Arany, Z., et al. 2005. Transcriptional coactivator PGC- $1 \alpha$ controls the energy state and contractile function of cardiac muscle. Cell Metab. 1:259-271.

58. Lehman, J.J., and Kelly, D.P. 2002. Gene regulatory mechanisms governing energy metabolism during cardiac hypertrophic growth. Heart Fail. Rev. 7:175-185.

59. Lehman, J.J., and Kelly, D.P. 2002. Transcriptional activation of energy metabolic switches in the developing and hypertrophied heart. Clin. Exp. Pharmacol. Physiol. 29:339-345.

60. Finck, B., Lehman, J.J., Barger, P.M., and Kelly, D.P. 2002. Regulatory networks controlling mitochondrial energy production in the developing, hypertrophied, and diabetic heart. In The cardiovascular system. B. Stillman and D.J. Stewart, editors. Cold Spring Harbor Laboratory Press. Cold Spring Harbor, New York, USA. 371-382.

61. Sano, M., et al. 2002. Activation and function of cyclin T-Cdk9 (positive transcription elongation factor-b) in cardiac muscle-cell hypertrophy. Nat. Med. 8:1310-1317.

62. Sano, M., et al. 2004. Activation of cardiac Cdk9 represses PGC-1 and confers a predisposition to heart failure. $E M B O J$. 23:3559-3569.

63. Huss, J.M., and Kelly, D.P. 2005. Mitochondrial energy metabolism in heart failure: a question of balance. J. Clin. Invest. 115:547-555. doi:10.1172/ JCI200524405.

64. Terada, S., Kawanaka, K., Goto, M., Shimokawa, T., and Tabata, I. 2005. Effects of high-intensity intermittent swimming on PGC- $1 \alpha$ protein expression in rat skeletal muscle. Acta Physiol. Scand. 184:59-65.

65. Taylor, E.B., et al. 2005. Endurance training increases skeletal muscle LKB1 and PGC- $1 \alpha$ protein abundance: effects of time and intensity. Am. J. Physiol. Endocrinol. Metab. 289:E960-E968.

66. Russell, A.P., et al. 2003. Endurance training in humans leads to fiber type-specific increases in levels of peroxisome proliferator-activated receptor- $\gamma$ coactivator- 1 and peroxisome proliferatoractivated receptor- $\alpha$ in skeletal muscle. Diabetes. 52:2874-2881.

67. Akimoto, T., et al. 2005. Exercise stimulates PGC-1 $\alpha$ transcription in skeletal muscle through activation of the p38 MAPK pathway. J. Biol. Chem. 280:19587-19593.

68. Lin, J., et al. 2002. Transcriptional co-activator 
PGC-1 $\alpha$ drives the formation of slow-twitch muscle fibers. Nature. 418:797-801.

69. Wende, A.R., Huss, J.M., Schaeffer, P.J., Giguère, V., and Kelly, D.P. 2005. PGC- $1 \alpha$ coactivates PDK4 gene expression via the orphan nuclear receptor ERR $\alpha$ : a mechanism for transcriptional control of muscle glucose metabolism. Mol. Cell. Biol. 25:10684-10694.

70. Yoon, J.C., et al. 2001. Control of hepatic gluconeogenesis through the transcriptional coactivator PGC-1. Nature. 413:131-138.

71. Koo, S.H., et al. 2004. PGC-1 promotes insulin resistance in liver through PPAR $\alpha$-dependent induction of TRB-3. Nat. Med. 10:530-534.

72. Bernal-Mizrachi, C., et al. 2003. Dexamethasone induction of hypertension and diabetes is PPAR-o dependent in LDL receptor-null mice. Nat. Med. 9:1069-1075.

73. Handschin, C., et al. 2005. Nutritional regulation of hepatic heme biosynthesis and porphyria through PGC-1 $\alpha$. Cell. 122:505-515.

74. Andrulionyte, L., Zacharova, J., Chiasson, J.L., and Laakso, M. 2004. Common polymorphisms of the PPAR- $\gamma 2$ (Pro12A1a) and PGC- $1 \alpha$ (Gly482Ser) genes are associated with the conversion from impaired glucose tolerance to type 2 diabetes in the STOP-NIDDM trial. Diabetologia. 47:2176-2184.

75. Ek, J., et al. 2001. Mutation analysis of peroxisome proliferator-activated receptor- $\gamma$ coactivator- 1 (PGC-1) and relationships of identified amino acid polymorphisms to type II diabetes mellitus. Diabetologia. 44:2220-2226.
76. Hara, K., et al. 2002. A genetic variation in the PGC-1 gene could confer insulin resistance and susceptibility to type II diabetes. Diabetologia. 45:740-743.

77. Kim, J.H., et al. 2005. Peroxisome proliferator-activated receptor $\gamma$ coactivator $1 \alpha$ promoter polymorphisms are associated with early-onset type 2 diabetes mellitus in the Korean population. Diabetologia. 48:1323-1330.

78. Kunej, T., Globocnik Petrovic, M., Dovc, P., Peterlin, B., and Petrovic, D. 2004. A Gly482Ser polymorphism of the peroxisome proliferator-activated receptor- $\gamma$ coactivator-1 (PGC-1) gene is associated with type 2 diabetes in Caucasians. Folia Biol. (Praha). 50:157-158.

79. Muller, Y.L., Bogardus, C., Pedersen, O., and Baier, L. 2003. A Gly482Ser missense mutation in the peroxisome proliferator-activated receptor $\gamma$ coactivator- 1 is associated with altered lipid oxidation and early insulin secretion in Pima Indians. Diabetes. 52:895-898.

80. Vimaleswaran, K.S., et al. 2005. Peroxisome proliferator-activated receptor- $\gamma$ co-activator- $1 \alpha$ (PGC$1 \alpha$ ) gene polymorphisms and their relationship to type 2 diabetes in Asian Indians. Diabet. Med. 22:1516-1521.

81. Oberkofler, H., et al. 2004. Complex haplotypes of the PGC-1 $\alpha$ gene are associated with carbohydrate metabolism and type 2 diabetes. Diabetes. 53:1385-1393.

82. Lacquemant, C., Chikri, M., Boutin, P., Samson, C., and Froguel, P. 2002. No association between the G482S polymorphism of the proliferator-activated receptor-gamma coactivator-1 (PGC-1) gene and type II diabetes in French Caucasians. Diabetologia. 45:602-603.

83. Stumvoll, M., et al. 2004. The Gly482Ser variant in the peroxisome proliferator-activated receptor gamma coactivator-1 is not associated with diabetes-related traits in non-diabetic German and Dutch populations. Exp. Clin. Endocrinol. Diabetes. 112:253-257.

84. Yoon, J.C., et al. 2003. Suppression of $\beta$ cell energy metabolism and insulin release by PGC-1 $1 \alpha$. Dev. Cell. 5:73-83.

85. Wilson, F.H., et al. 2004. A cluster of metabolic defects caused by mutation in a mitochondrial tRNA. Science. 306:1190-1194.

86. Attie, A.D., and Kendziorski, C.M. 2003. PGC-1 $\alpha$ at the crossroads of type 2 diabetes. Nat. Genet. 34:244-245.

87. Mootha, V.K., et al. 2003. PGC- $1 \alpha$-responsive genes involved in oxidative phosphorylation are coordinately downregulated in human diabetes. Nat. Genet. 34:267-273.

88. Patti, M.E., et al. 2003. Coordinated reduction of genes of oxidative metabolism in humans with insulin resistance and diabetes: potential role of PGC1 and NRF1. Proc. Natl. Acad. Sci. U. S. A 100:8466-8471.

89. Morino, K., et al. 2005. Reduced mitochondrial density and increased IRS- 1 serine phosphorylation in muscle of insulin-resistant offspring of type 2 diabetic parents. J. Clin. Invest. 115:3587-3593. doi:10.1172/JCI25151. 